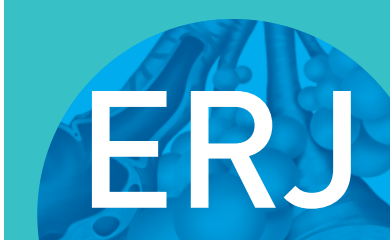

open research

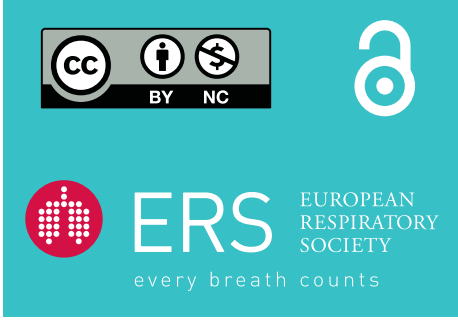

\section{A sputum sample processing method for community and mobile tuberculosis diagnosis using the Xpert MTB/RIF assay}

\section{To the Editor:}

Tuberculosis (TB) remains a global public health emergency and claims the lives of more people annually than any other infectious disease. TB disproportionately affects people living in poverty, with $95 \%$ of cases and $98 \%$ of deaths occurring in developing countries [1]. In high income countries, $\mathrm{TB}$ is now concentrated in populations who originate from high burden countries and in socially vulnerable and excluded groups, including homeless people, drug and alcohol users and prisoners. These populations often experience poor access to health services and are at increased risk of delayed diagnosis, onward transmission, drug resistance, severe disease and preventable death.

Despite a decline in reported TB cases since 2011, London accounted for 2210 of the 5664 cases reported in England in 2016; this amounts to $40 \%$ of all cases reported nationally and equates to more TB cases than the Netherlands (889 cases), Greece (443), Norway (299) and Denmark (330) combined [2]. The recent decline in cases has not been seen among homeless and vulnerable populations who now account for one in six of all TB cases reported in London, where the numbers of people sleeping rough has more than doubled since 2010 [3]. TB patients in London with social risk factors are more likely to be infectious, have drug-resistant TB and not complete treatment, and homelessness is an independent risk factor for multidrug-resistant TB [4].

Active case finding using targeted mobile digital radiology is widely recommended and used in high income countries to reduce diagnostic delays and prevent onward transmission. Find\&Treat are a specialist outreach team dedicated to the early detection and management of TB and blood-borne viral disease among homeless and vulnerable people. The team operate a Mobile Health Unit (MHU) routinely targeting homeless hostels, day centres, street kitchens and drug treatment services in London, and throughout the UK, in response to TB incidents and outbreaks. The MHU is equipped to provide digital chest radiographs, which are interpreted on the spot by experienced reporting radiographers, to identify abnormalities consistent with active pulmonary TB. Radiography alone is not diagnostic for pulmonary TB and patients with abnormal chest radiographs are required to provide sputum samples or attend local healthcare facilities for further investigations. This delay in diagnosis confirmation increases the risk of vulnerable patients disengaging from services and becoming lost to follow-up care. The Xpert MTB/RIF assay (Cepheid, Sunnyvale, CA, USA) is a nucleic acid amplification test that simultaneously detects Mycobacterium tuberculosis complex and markers of rifampicin resistance in less than $2 \mathrm{~h}$. This test has the potential to allow patients with abnormal chest radiographs to be investigated in the community. However, roll-out beyond laboratory settings in high income countries has been limited by health and safety considerations regarding sputum sample handling. To overcome this, the Find\&Treat service sourced and utilised a "septum sample pot", which allows samples to be treated and processed safely on the MHU. Ethical approval for this study was granted by the NRES Committee East of England - Essex, Research Ethics Committee (On March 20, 2014; reference: 10/H0302/51).

@ERSpublications

The Xpert MTB/RIF assay can rapidly diagnose tuberculosis, but sputum samples cannot be safely processed unless in a lab. The septum sample pot allows safe handling of sputum and has allowed a mobile TB unit to run the assay in community settings. http://ow.ly/HOA130mS6LG

Cite this article as: Gliddon HD, Shorten RJ, Hayward AC, et al. A sputum sample processing method for community and mobile tuberculosis diagnosis using the Xpert MTB/RIF assay. ERJ Open Res 2019; 5: 00165-2018 [https://doi.org/10.1183/23120541.00165-2018].

Copyright $\odot$ ERS 2019. This article is open access and distributed under the terms of the Creative Commons Attribution Non-Commercial Licence 4.0. 
The MHU is equipped with a Gene Xpert IV system (Cepheid) to run the Xpert MTB/RIF assay as a point-of-care (POC) test. The high sensitivity and specificity of the assay has been extensively studied and it is now recommended by the World Health Organization for TB diagnosis [5, 6]. Following addition of the sample to a cartridge, the assay is entirely automated. One of the major barriers to the roll-out of the Xpert MTB/RIF assay in the community is the biosafety requirements of adding the sample reagent to the sputum sample, vortexing or shaking vigorously (and so potentially producing aerosolised droplet nuclei of $M$. tuberculosis) and then adding the processed sample to the cartridge. This is typically performed in a microbiological safety cabinet, which is not available to the staff of the MHU. To overcome the risk of producing aerosolised droplet nuclei, patients with abnormal chest radiographs are given a "septum sample pot" (figure 1), which comprises a container with a rubber VACUETTE valve stopper (product number 724300, Urine Beaker with Safety Stopper $100 \mathrm{~mL}$, white lid, single-packed, sterile; Greiner Bio-One GmbH, Kremsmünster, Austria). Patients step off the MHU to produce a sputum sample in the

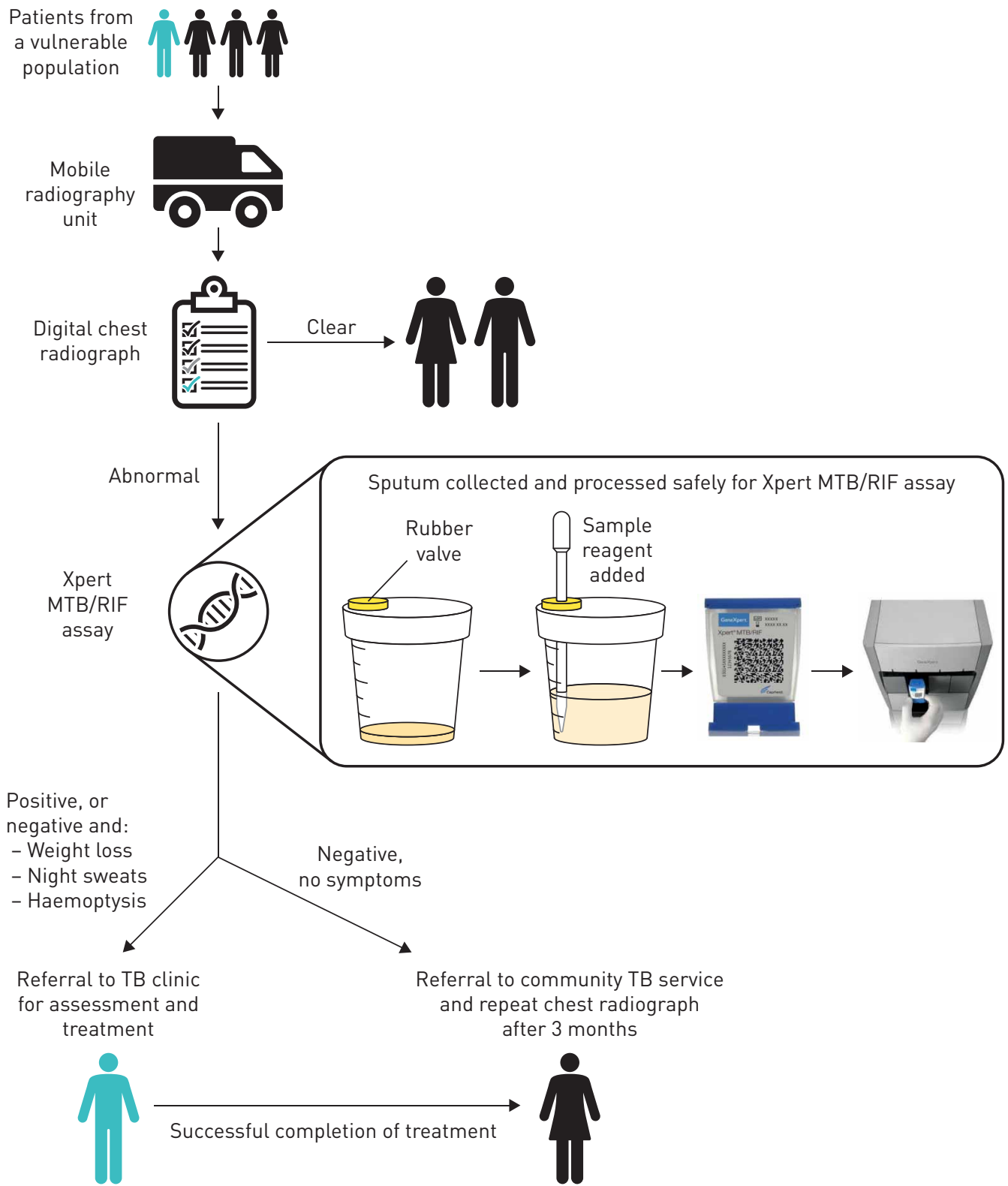

FIGURE 1 Patient pathway for the diagnosis of pulmonary tuberculosis (TB) on the mobile health unit. Patients from a vulnerable population are first offered a digital chest radiograph. If indicative of a potential pulmonary TB infection, a sputum sample is collected in a secure septum sample pot for processing before performing the Xpert MTB/RIF assay. The rubber valve stopper in the lid of the septum sample pot allows access with a pipette, without needing to open the lid and manipulate the sample. Patients are referred to TB clinics if the Xpert MTB/RIF assay result is positive, or if they display symptoms typical of TB. 
open air and away from other people to avoid possible transmission of M. tuberculosis or other airborne pathogens. The lid is fastened by the patient once the sputum sample has been added and can then be safely transferred to staff on the MHU. The Xpert MTB/RIF sample reagent is then added with a pipette that enters through the valve (septum) on the lid, the pipette is then removed, disposed of appropriately, and the septum self-seals allowing the sample and reagent to be mixed through vigorous shaking. This sample reagent lyses $M$. tuberculosis and therefore makes the sample safe to work with. Studies have shown that when live M. tuberculosis cultures were added to fresh sputum samples and treated with the sample reagent in a 1:2 ratio, an 8-log decrease in viability was observed following an incubation time of $15 \mathrm{~min}$ [7]. The processed sample can then be retrieved using a separate pipette, again through the septum valve, and added to the Xpert cartridge. Training of MHU personnel covers the need for an adequate sputum sample volume (minimum $2 \mathrm{~mL}$ ). Staff can estimate the volume of sputum visually and are trained to add 2-3 times this volume of sample reagent using a plastic pipette via the rubber septum. In addition, staff assess the quality of sputum and reject salivary samples that may provide false negative results.

Prior to the implementation of the Xpert MTB/RIF assay, a TB nurse specialist or outreach worker accompanied patients with suspected pulmonary TB to local hospitals for assessment, conventional microbiological investigation and possible admission. The MHU screens between 8000-10000 patients annually. Approximately $1 \%$ of patients screened have abnormal chest radiology suggestive of active TB; however, approximately $75 \%$ of referred patients are subsequently diagnosed as negative for active TB. The resource implications of referrals, including staff time during the accompanied visit, administration, clinician time, and urgent pathology samples sent to the laboratories is significant. In addition, the inconvenience to patients is considerable and many are reluctant to go to hospital. The aims of implementing Xpert MTB/RIF on the MHU are to: 1) rapidly identify cases of active TB, with accompanied referral to link them to care and commence treatment the same day; 2) reduce the risk of patients disengaging from services and becoming lost to follow-up; and 3) prevent unnecessary referrals to secondary care. The new pathway using the Xpert MTB/RIF assay for patients with radiological abnormalities (figure 1) has been well received by staff and patients. In addition to enabling simple and safe POC diagnosis, this pathway exploits the high negative predictive value of Xpert MTB/RIF [8]. Patients able to produce a voluntary sputum sample who have a negative Xpert MTB/RIF result can be assessed as presenting an extremely low transmission risk and be further investigated for TB or other respiratory disease in the community, including patients with a false negative Xpert MTB/RIF result who are more likely to be smear negative and therefore less likely to be infectious [9].

This novel approach enables safe processing of sputum specimens in the community, without the need for containment facilities or a microbiological safety cabinet. We believe this is the first example of a molecular diagnostic test for TB being used in the community at the POC for socially vulnerable and excluded TB patients in Europe. This affordable technology has the potential to improve biosafety measures in high TB burden countries where GeneXpert is often used outside laboratory settings in areas where containment measures are not adopted for direct Xpert testing and achieving adequate ventilation for safe handling of specimens may be challenging [10]. New smaller, portable, battery-powered devices that run one GeneXpert cartridge (i.e. the GeneXpert Omni [11]) will further enable POC TB molecular diagnosis to be performed in other settings where rapid confirmation of pulmonary disease is highly advantageous and barriers to further diagnostic testing are operationally and financially challenging, such as in prisons and custodial settings.

Harriet D. Gliddon ${ }^{1}$, Robert J. Shorten ${ }^{2,3}$, Andrew C. Hayward ${ }^{4}$ and Alistair Story ${ }^{5}$

${ }^{1}$ London Centre for Nanotechnology, University College London, London, UK. ${ }^{2}$ Public Health England, Public Health Laboratory Manchester, Manchester Royal Infirmary, Manchester, UK. ${ }^{3}$ Dept of Infection, Centre for Clinical Microbiology, University College London, London, UK. ${ }^{4}$ University College London, Institute of Epidemiology and Health Care, London, UK. ${ }^{5}$ Find\&Treat, University College London Hospitals, London, UK.

Correspondence: Alistair Story, Find\&Treat, University College London Hospitals NHS Foundation Trust, 4th Floor East, 250 Euston Rd, London, NW1 2PG, UK. E-mail: al.story@nhs.net

Received: Sept 192018 | Accepted after revision: Nov 072018

Acknowledgements: The authors would like to thank UCLH for funding the MHU and Sushma Patel (Cepheid) for sourcing the sample pots.

Conflict of interest: H.D. Gliddon has nothing to disclose. R.J. Shorten reports personal fees from Cepheid (sponsored attendance at an international conference in 2016), outside the submitted work. A.C. Hayward has nothing to disclose. A. Story has nothing to disclose. 
Support statement: This service development research was funded through NIHR Programme Grant RP-PG-0407-10340. H.D. Gliddon acknowledges support from the i-sense Engineering and Physical Sciences Research Council (EPSRC) IRC in Early Warning Sensing Systems for Infectious Diseases (EP/K031953/1; www.i-sense.org.uk). Funding information for this article has been deposited with the Crossref Funder Registry.

\section{References}

1 Benatar SR, Upshur R. Tuberculosis and poverty: what could (and should) be done? Int J Tuberc Lung Dis 2010; 14: 1215-1221.

2 European Centre for Disease Control and Prevention/WHO Regional Office for Europe. Tuberculosis surveillance and monitoring in Europe 2018, 2016 data. Stockholm, European Centre for Disease Prevention and Control, 2018. https://ecdc.europa.eu/sites/portal/files/documents/ecdc-tuberculosis-surveillance-monitoring-Europe-201819mar2018.pdf Date last accessed: July 30, 2018.

3 Ministry of Housing, Communities and Local Government. Rough Sleeping Statistics Autumn 2017, England. London, Ministry of Housing, Communities and Local Government, 2017. https://assets.publishing.service.gov.uk/ government/uploads/system/uploads/attachment_data/file/682001/Rough_Sleeping_Autumn_2017_Statistical_Release__revised.pdf Date last accessed: July 29, 2018. Date last updated: February 16, 2018.

4 Public Health England. Tackling tuberculosis in under-served populations: a resource for TB control boards and their partners. London, PHE 2017. https://assets.publishing.service.gov.uk/government/uploads/system/uploads/ attachment_data/file/592274/Tackling_TB_in_Under-Served_Populations_-_A_Resource_for_TBCBs_and_partners. pdf Date last accessed: June 29, 2018

5 Steingart KR, Schiller I, Horne DJ, et al. Xpert ${ }^{\circ}$ MTB/RIF assay for pulmonary tuberculosis and rifampicin resistance in adults. Cochrane Database Syst Rev 2014: 1: CD009593.

6 Boehme CC, Nicol MP, Nabeta P, et al. Feasibility, diagnostic accuracy, and effectiveness of decentralised use of the Xpert MTB/RIF test for diagnosis of tuberculosis and multidrug resistance: a multicentre implementation study. Lancet 2011; 377: 1495-1505.

7 Helb D, Jones M, Story E, et al. Rapid detection of Mycobacterium tuberculosis and rifampin resistance by use of on-demand, near-patient technology. J Clin Microbiol 2010; 48: 229-237.

8 Zeka AN, Tasbakan S, Cavusoglu C. Evaluation of the GeneXpert MTB/RIF assay for rapid diagnosis of tuberculosis and detection of rifampin resistance in pulmonary and extrapulmonary specimens. J Clin Microbiol 2011; 49: 4138-4141.

9 Opota O, Senn L, Prod'hom G, et al. Added value of molecular assay Xpert MTB/RIF compared to sputum smear microscopy to assess the risk of tuberculosis transmission in a low-prevalence country. Clin Microbiol Infect 2016 22: 613-619.

10 World Health Organization. Tuberculosis Laboratory Safety Manual. Geneva, World Health Organization, 2012. http://apps.who.int/iris/bitstream/handle/10665/77949/9789241504638_eng.pdf;jsessionid=A248722DEDD0D0C0B6 ABC546AA389ADF?sequence=1 Date last accessed: September 11, 2018.

11 Drain PK, Garrett NJ. The arrival of a true point-of-care molecular assay-ready for global implementation? Lancet Glob Health 2015; 3: e663-e664. 